\title{
Eigen-Based Clutter Filter Design for Ultrasound Color Flow Imaging: A Review
}

\author{
Alfred C. H. Yu, Member, IEEE, and Lasse Lovstakken, Member, IEEE
}

\begin{abstract}
Proper suppression of tissue clutter is a prerequisite for visualizing flow accurately in ultrasound color flow imaging. Among various clutter suppression methods, the eigen-based filter has shown potential because it can theoretically adapt its stopband to the actual clutter characteristics even when tissue motion is present. This paper presents a formative review on how eigen-based filters should be designed to improve their practical efficacy in adaptively suppressing clutter without affecting the blood flow echoes. Our review is centered around a comparative assessment of two eigen-filter design considerations: 1) eigen-component estimation approach (single-ensemble vs. multi-ensemble formulations), and 2) filter order selection mechanism (eigenvalue-based vs. frequencybased algorithms). To evaluate the practical efficacy of existing eigen-filter designs, we analyzed their clutter suppression level in two in vivo scenarios with substantial tissue motion (intra-operative coronary imaging and thyroid imaging). Our analysis shows that, as compared with polynomial regression filters (with or without instantaneous clutter downmixing), eigen-filters that use a frequency-based algorithm for filter order selection generally give Doppler power images with better contrast between blood and tissue regions. Results also suggest that both multi-ensemble and single-ensemble eigen-estimation approaches have their own advantages and weaknesses in different imaging scenarios. It may be beneficial to develop an algorithmic way of defining the eigen-filter formulation so that its performance advantages can be better realized.
\end{abstract}

\section{INTRODUCTION}

A $\mathrm{N}$ important step in the processing of color flow imaging (CFI) data is the suppression of slow-time clutter originating from tissue reverberations and beam side lobe leakages. If done properly, this clutter filtering step can improve the flow visualization performance by reducing the amount of false coloring seen in the tissue regions of CFI maps and helping to lower the bias of velocity estimates seen in the blood regions. In early CFI work, clutter was suppressed via high-pass filtering of the slow-time ensemble at each sample volume (with the assumption that clutter correspond to low-frequency contents because tissues are relatively stationary) [1]. However, because the stopband of these high-pass filters are not adaptive to the actual clutter characteristics, they tend to be less effective when there are substantial tissue movements that give rise

Manuscript received August 25, 2009; accepted January 20, 2010. The authors have contributed equally to the preparation of this article.

A. C. H. Yu is with the Medical Engineering Program, The University of Hong Kong, Pokfulam, Hong Kong SAR (e-mail: alfred.yu@hku.hk).

L. Lovstakken is with the Department of Circulation and Medical Imaging, The Norwegian University of Science and Technology, Trondheim, Norway. He is also affiliated with Trondheim University Hospital (e-mail: lasse.lovstakken@ntnu.no).

Digital Object Identifier 10.1109/TUFFC.2010.1521 to clutter with nonzero Doppler frequency contents. In fact, the use of high-pass filters in CFI data processing is not trivial because the limited number of slow-time data samples available ( 8 to 16 per sample volume, as limited by real-time constraints) makes it challenging for conventional digital filters to effectively suppress clutter without distorting the desired blood echoes [2]

To better address the clutter filtering problem in CFI, studies have proposed more advanced filtering methods that can adapt their stopband to the actual clutter contents. Among these reported methods, it has been suggested that the eigen-based filter has potential to adaptively suppress slow-time clutter with variable spectral characteristics [3]. This approach works by directly removing eigen-components (or orthogonal bases) that represent clutter in the slow-time signal. Its attenuation response is, in theory, adaptive to clutter contents because the eigencomponents are estimated based on the actual slow-time signal statistics. In the context of CFI, eigen-based processing was first considered by Allam et al. [4] and Vaitkus et al. [5], who adopted an eigen-based estimation framework called multiple signal classification (MUSIC) that is used in array processing. Around the same time, Ledoux et al. [6] developed an eigen-filtering framework that works via singular value decomposition (SVD). Bjaerum et al. [7], Kargel et al. [8], and Song et al. [9] also reported a similar eigen-filtering strategy that is based on eigenvalue decomposition (EVD), and Kruse and Ferrara [10] presented a slightly modified eigen-filtering framework to suppress clutter in the slow-time ensembles acquired from a swept-scan imaging mode. Using similar concepts, Gallippi and Trahey [11] proposed an independent component analysis approach for computing the signal decomposition, whereas Li et al. [12] considered performing this task using sparse component analysis. Recently, Lovstakken et al. [13] and You and Wang [14] addressed the computational issues in using eigen-filters for CFI and reported a more refined filter formulation. Also, Yu and Cobbold [15] suggested another eigen-filtering method that is based on a Hankel-SVD formulation.

Although many studies have been devoted to the use of eigen-based filters in CFI applications, there are two major design considerations that have not been well investigated. First, it remains unclear as to which estimation methodology is more suitable for accurately finding the eigen-components of a slow-time ensemble. Another issue that needs further attention is the algorithm used to select the eigen-filter order (i.e., the number of eigen-components to remove from the original signal). If the filter order is chosen incorrectly, clutter may not be sufficiently attenu- 
ated, or parts of the blood signal may be concomitantly removed in the filtering process.

The overall goal of this work is to perform a formative evaluation of how eigen-based clutter filters should be designed to realize its adaptive filtering potential in CFI data processing. We shall place emphasis on the two major design considerations related to eigen-based clutter suppression: eigen-component estimation approach and filter order selection method. The analysis will begin from a theoretical standpoint where we examine the advantages and limitations of different eigen-filter formulations. This discussion will be followed by an experimental analysis that examines the efficacy of various eigen-filter formulations in processing raw data sets acquired in vivo from CFI scenarios known to be challenging to clinicians.

\section{BACKGROUND THEORY}

\section{A. Slow-Time Signal Model}

To facilitate description of eigen-based clutter filter design, we first consider the slow-time signal model for a CFI sample volume that encompasses blood flow. In this case, the slow-time signal generally comprises of three constituent components: 1) blood echoes, as returned from moving red cell scatterers; 2) clutter, which arises from reverberations and beam side lobes covering tissue regions; 3) random white noise, which originates from thermal and electronic fluctuations. In contrast, for a CFI sample volume nominally located in tissue regions, its slow-time signal should only comprise of tissue clutter and random white noise (because blood flow is not present). As such, a slowtime ensemble $\mathbf{x}$ can generally be expressed in the following two forms for a given ensemble size $N_{D}$ sampled at the pulse repetition frequency $\mathrm{PRF}$ :

$$
x=\left[\begin{array}{c}
x(0) \\
x(1) \\
\vdots \\
x\left(N_{D}-1\right)
\end{array}\right]= \begin{cases}\mathbf{b}+\mathbf{c}+\mathbf{n} & (\text { vasculature }) \\
\mathbf{c}+\mathbf{n} & (\text { tissue })\end{cases}
$$

In (1), $x(n)$ represents the $n$th slow-time data sample, and b, c, and $\mathbf{n}$ are vectors of length $N_{D}$ for blood echoes, clutter, and white noise, respectively. To determine whether blood signal is present in the slow-time ensemble of a sample volume, it is necessary to first suppress the clutter component, the strength of which is usually much higher (over $20 \mathrm{~dB}$ ) than the other slow-time signal components. Such an objective is central to the design of clutter filters.

\section{B. Basic Principles of Eigen-Based Clutter Filters}

A conceptual illustration of the eigen-based filter is shown in Fig. 1. This filtering strategy generally begins by decomposing a sample volume's slow-time signal contents

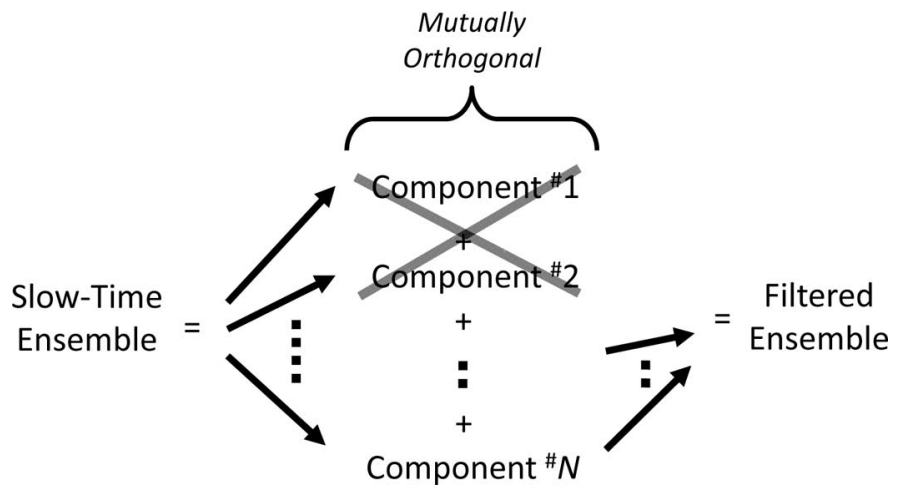

Fig. 1. Conceptual illustration of the principles behind eigen-based clutter filters. During the filtering process, eigen-components (often the more dominant ones) that represent clutter are removed.

into a sum of mutually orthogonal components that have the minimum mean-squared modeling error [6]-[9]. Such a decomposition, often referred to as the Karhunen-Loeve expansion, can be mathematically expressed as follows for a given slow-time ensemble vector $\mathbf{x}$ :

$$
\mathbf{x} \approx \sum_{k} \gamma_{k} \mathbf{e}_{k}
$$

In (2), $\mathbf{e}_{k}$ is the $k$ th eigenvector (of length $N_{D}$ ) and $\gamma_{k}$ is the corresponding expansion weight that satisfies the following orthogonality relation:

$$
E\left\{\left(\gamma_{k} \mathbf{e}_{k}\right)^{* T}\left(\gamma_{l} \mathbf{e}_{l}\right)\right\}= \begin{cases}\lambda_{k} & (k=l) \\ 0 & (k \neq l)\end{cases}
$$

where $\lambda_{k}$ is referred to as the $k$ th eigenvalue. By definition, the eigen-components in (2) are ordered in a descending energy order, and hence $\lambda_{k} \mathbf{e}_{k}$ can be considered as the $k$ th -largest eigen-component. The goal of the eigen-based filter is to then identify and remove eigen-components that represent clutter in the slow-time signal. Assuming that $K_{\mathrm{c}}$ eigen-components correspond to clutter, the eigenbased filter can be interpreted as having a filter order of $\left(K_{\mathrm{c}}-1\right)$. This clutter suppression approach is inherently adaptive to the slow-time ensemble characteristics because its attenuation response is defined according to the eigencomponents in the slow-time signal composition.

\section{The First Stage of Eigen-Filters: Eigen- Component Estimation}

\section{A. Overview of Computation Approach}

The common way of finding the eigen-components in (2) is to compute the eigenvalue decomposition (EVD) of the slow-time signal's correlation matrix (i.e., $E\left\{\mathbf{x x}^{*} T\right\}$, or the expected outer product between the signal vector and its conjugate transpose). This computation approach stems from matrix algebra's version of the spectral theorem [16], which relates the slow-time correlation matrix $\mathbf{R}$ 
to the eigen-components in (2), based on the orthogonality principle as follows:

$$
\mathbf{R}=E\left\{\mathbf{x x}^{{ }^{*} T}\right\}=\sum_{k} \lambda_{k} \mathbf{e}_{k} \mathbf{e}_{k}^{*_{T}} .
$$

Because the correlation matrix in (4) has a self-adjoint structure (i.e., $\mathbf{R}=\mathbf{R}^{* T}$ ), it is possible to define a nonsquare data matrix $\mathbf{A}$ that satisfies the relation $\mathbf{R}=$ $\mathbf{A A}^{*} T$. It follows that the singular value decomposition (SVD) of $\mathbf{A}$ is connected to the EVD of $\mathbf{R}$ according to the following duality relation:

$$
\mathbf{A}=\sum_{k} \sigma_{k} \mathbf{u}_{k} \mathbf{v}_{k}^{* T} \Leftrightarrow \mathbf{A} \mathbf{A}^{* T}=\sum_{k} \sigma_{k}^{2} \mathbf{u}_{k} \mathbf{u}_{k}^{* T} \equiv \mathbf{R},
$$

where $\sigma_{k}$ is the $k$ th singular value with $\mathbf{u}_{k}$ and $\mathbf{v}_{k}$ as the corresponding left/right singular vectors. Comparing (4) and (5), it can be seen that: 1) the eigenvectors in the EVD of $\mathbf{R}$ are equivalent to the left singular vectors in the SVD of $\mathbf{A}$, and 2) each eigenvalue is essentially the square of the corresponding singular value. These two properties show that both the SVD of $\mathbf{A}$ and the EVD of $\mathbf{R}$ may be used to calculate the eigen-components in (2). Note that EVD/SVD can be solved using numerical algorithms such as power iterations and QR factorization, which generally involve a cubic-order number of floating point operations (flops) [16].

To carry out the above eigen-computation approaches in practice, it is necessary to first estimate the slow-time correlation matrix $\mathbf{R}$ or the data matrix A. As reported in existing eigen-filter designs, this estimation process can be done based on formulations that involve either multiple slow-time ensembles or a single slow-time ensemble [17]. The principles of both formulations and their suitability in slow-time clutter filtering will be discussed in the following subsections.

\section{B. Multi-Ensemble-Based Formulation}

One method of estimating the slow-time correlation matrix during operations is to first create a data matrix by stacking together multiple realizations of slow-time ensembles [see Fig. 2(a)] and then compute the outer product between the data matrix and its conjugated transpose. For a set of $M$ slow-time ensembles, this formulation would give the following forms of the data matrix $\mathbf{A}$ (with size $N_{D} \times M$ ) [6] and the correlation matrix $\mathbf{R}$ (with size $\left.N_{D} \times N_{D}\right)[7]-[9]:$

$$
\begin{aligned}
& \mathbf{A}=\frac{1}{\sqrt{M}}\left[\begin{array}{cccc}
\mid & \mid & & \mid \\
x_{1} & x_{2} & \cdots & x_{M} \\
\mid & \mid & & \mid
\end{array}\right], \\
& \mathbf{R}=\mathbf{A A}^{{ }^{*} T}=\frac{1}{M} \sum_{m=1}^{M} \mathbf{x}_{m} \mathbf{x}_{m}^{{ }^{*} T},
\end{aligned}
$$

where $x_{m}$ is the $m$ th realization in the ensemble set. Note that the SVD/EVD computed from these two matrices

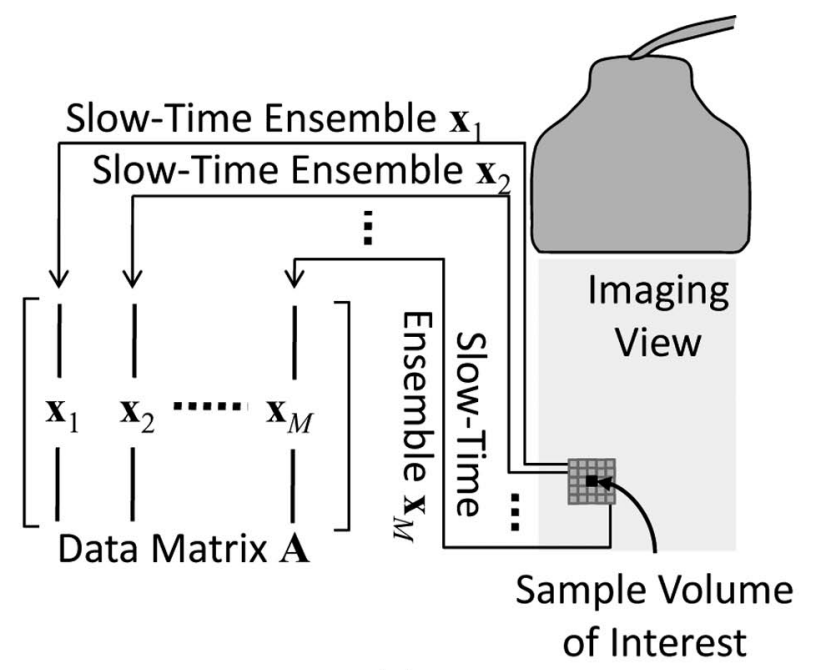

(a)

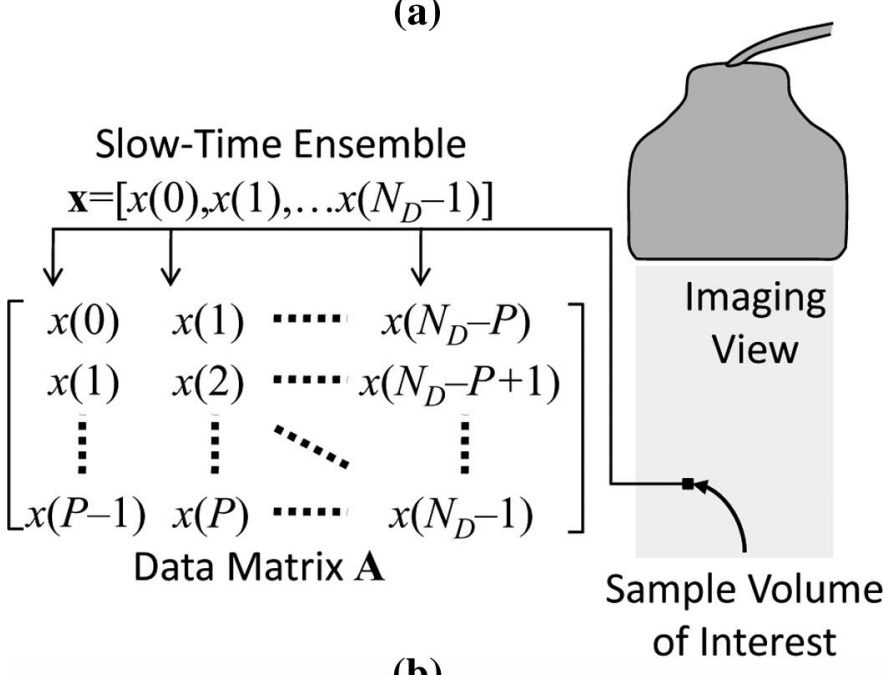

(b)

Fig. 2. Principles of two different eigen-component estimation methods: (a) a multi-ensemble approach that uses a set of slow-time ensembles within a spatial window; (b) a single-ensemble approach that involves redundant use of slow-time data samples.

would comprise $N_{D}$ eigen-components if (6) and (7) have full matrix rank (i.e., linear independence between all rows), which can be achieved when the number of independent slow-time ensembles is at least equal to the ensemble size (i.e., $M \geq N_{D}$ ). Nevertheless, the ensemble set used to form (6) and (7) is required to possess similar clutter statistics because these matrices are formed via ensemble stacking and averaging. In the literature, it was suggested that the ensemble set can be chosen either from CFI sample volumes along the same beam line [6]-[11] or from ones within a spatial window centered about the sample volume concerned [13]. The latter way, which is illustrated in Fig. 2(a), seems more appropriate because adjacent CFI sample volumes within an imaging view are more likely to share similar clutter statistics.

For the multi-ensemble eigen-estimation approach, clutter suppression is often achieved via a linear regression strategy that computes the fitting residual between the clutter eigenvectors and the slow-time ensemble of in- 
terest. From a vector space perspective [16], this regression task is the same as a least-squares projection of the slow-time signal vector onto the orthogonal equivalent of the clutter model subspace. Assuming that there are $K_{\mathrm{c}}$ eigen-components corresponding to clutter and denoting this subset as $\boldsymbol{\Phi}_{\mathrm{c}}$, the filtered slow-time ensemble $\mathbf{y}$ would be equal to the following matrix product between the raw ensemble $\mathbf{x}$ and the orthogonal complement of all the clutter eigenvectors:

$$
\mathbf{y}=\left(\mathbf{I}-\sum_{k=1}^{K_{\mathrm{c}}} \mathbf{e}_{k} \mathbf{e}_{k}^{* T}\right) \mathbf{x}, \quad \mathbf{e}_{k} \in \boldsymbol{\Phi}_{\mathrm{c}}
$$

From the filtered signal vector, we can then obtain the blood power and velocity estimates respectively via the mean-squared sum of filtered samples and the lag-one autocorrelation phase [18]. Note that, for the multi-ensemble approach, slow-time clutter is usually not filtered via direct subtraction of clutter eigen-components from the concerned sample volume's slow-time ensemble. This more direct way of removing clutter is not suitable for the multi-ensemble formulation because there are inherent magnitude differences between the concerned CFI sample volume's actual signal contents and the eigen-components that are computed over a broader spatial window (i.e., spatially averaged eigen-component estimates).

\section{Single-Ensemble-Based Formulation}

Instead of relying on multiple ensembles, it is possible to use a single slow-time ensemble to form the matrices A and R. In this second formulation, the slow-time data matrix is created by dividing a slow-time ensemble into lag-one overlapping subsets and stacking the subsets together in different columns [see Fig. 2(b)]. The resulting data matrix would take on the following Hankel structure (i.e., with constant reverse diagonals) [15]:

$$
\mathbf{A}=\frac{1}{\sqrt{P}}\left[\begin{array}{cccc}
x(0) & x(1) & \cdots & x\left(N_{D}-P\right) \\
x(1) & x(2) & \cdots & x\left(N_{D}-P+1\right) \\
\vdots & \vdots & \ddots & \vdots \\
x(P-1) & x(P) & \cdots & x\left(N_{D}-1\right)
\end{array}\right]
$$

where $P$ is a dimension parameter representing the subset size, and it is often set to ceil $\left(N_{D} / 2\right)$ to form the most subsets from a slow-time ensemble. From the $P \times\left(N_{D}-\right.$ $P+1$ ) data matrix defined in (9), a slow-time correlation matrix of size $P \times P$ can then be computed as

$$
\begin{aligned}
\mathbf{R} & =\mathbf{A A}^{* T} \\
& =\left[\begin{array}{cccc}
R_{0}(0) & R_{1}(-1) & \cdots & R_{P-1}(-P+1) \\
R_{0}(1) & R_{1}(0) & \cdots & R_{P-1}(-P+2) \\
\vdots & \vdots & \ddots & \vdots \\
R_{0}(P-1) & R_{1}(P-2) & \cdots & R_{P-1}(0)
\end{array}\right],
\end{aligned}
$$

with $R_{k}(l)$ being the $l$ th-lag autocorrelation estimate found from the following average of correlation values over an ensemble subset of $N_{D}-P+1$ samples (with $k$ as the first sample index at lag-zero):

$$
R_{k}(l)=\frac{1}{P} \sum_{n=k}^{k+N_{D}-P} x(n+l) x^{*}(n) .
$$

Note that (9) and (10) theoretically assume statistical stationarity between samples in the slow-time ensemble because of the data redundancy introduced in the matrix structures. Also, their respective SVD/EVD would give $P$ eigen-components with size- $P$ vectors. Because $P=$ $\operatorname{ceil}\left(N_{D} / 2\right)$ in most cases, as mentioned previously, the maximum number and size of eigen-components obtainable from this eigen-estimation method are both equal to ceil $\left(N_{D} / 2\right)$, or half the amount available from the multiensemble formulation. To reconstruct eigen-components with size- $N_{D}$ vectors, we can first create a rank-one Hankel matrix from each singular vector pair's outer product $\mathbf{u}_{k} \mathbf{v}_{k}^{* T}$ and then average along the $N_{D}$ reverse diagonals of this matrix [15].

For the single-ensemble eigen-estimation approach, we can obtain the filtered signal vector through direct subtraction of the clutter eigen-components from the raw ensemble. In other words, the filtered slow-time ensemble is simply equal to the sum of the non-clutter eigen-components. For this way of obtaining the filtered signal, it is actually possible to obtain blood power and velocity estimates directly from the eigen-component characteristics (i.e., without reconstructing the filtered signal). In particular, because the sum of eigenvalues is equal to the signal energy [16], the average blood power can be found by adding together the eigenvalues for the non-clutter eigen-components as follows:

$$
\rho_{\mathbf{y}}=\frac{1}{P} \sum_{k=K_{\mathrm{c}}+1}^{P} \lambda_{k}
$$

The mean blood velocity estimate can be found from the lag-one autocorrelation phase [18] of the non-clutter eigencomponents.

\section{Comparison of Formulations}

Table I summarizes the theoretical differences between the multi-ensemble and single-ensemble eigen-estimation approaches and it compares their pros and cons. As can be seen, the two approaches differ in at least four aspects: 1) the set of slow-time data involved in the eigen-estimation process, 2) the number of eigen-components computed, 3 ) the statistical assumption about the slow-time data characteristics, and 4) the estimation strategy for filtered power and flow velocity. For the multi-ensemble approach, the eigen-components are computed from a spatial average of slow-time ensembles. These eigen-component estimates are consistent as long as the spatial window of slow-time ensemble realizations has uniform clutter sta- 
TABle I. Theoretical Comparison of Eigen-Estimation Approaches.

\begin{tabular}{|c|c|c|}
\hline Attribute & Multi-Ensemble Formulation & Single-Ensemble Formulation \\
\hline $\begin{array}{l}\text { Data involved in eigen- } \\
\text { estimation }\end{array}$ & $\begin{array}{l}\text { The slow-time ensemble from } M \text { spatial } \\
\text { locations, usually taken from a window } \\
\text { centered about the sample volume concerned }\end{array}$ & $\begin{array}{l}\text { Only the slow-time ensemble at } \\
\text { the sample volume concerned }\end{array}$ \\
\hline $\begin{array}{l}\text { Number of eigen- } \\
\text { components available }\end{array}$ & $N_{D}\left(\right.$ if $\left.M \geq N_{D}\right)$ & $P\left(\right.$ at most $\left.\operatorname{ceil}\left(N_{D} / 2\right)\right)$ \\
\hline Statistical assumption & $\begin{array}{l}\text { All } M \text { realizations share similar clutter } \\
\text { statistics (i.e., spatial stationarity) }\end{array}$ & $\begin{array}{l}\text { Data samples within ensemble } \\
\text { share similar clutter statistics } \\
\text { (i.e., slow-time stationarity) }\end{array}$ \\
\hline Advantages & $\begin{array}{l}\text { Eigen-components can take on any arbitrary } \\
\text { form based on ensemble characteristics }\end{array}$ & $\begin{array}{l}\text { Only involve slow-time ensemble } \\
\text { of concerned sample volume }\end{array}$ \\
\hline \multirow[t]{2}{*}{ Limitations } & $\begin{array}{l}\text { 1) Requires at least } N_{D} \text { realizations of } \\
\text { slow-time ensembles for consistent eigen- } \\
\text { component estimation }\end{array}$ & $\begin{array}{l}\text { 1) Can obtain at most half the } \\
\text { number of eigen-components } \\
\text { available from multi-ensemble } \\
\text { formulation }\end{array}$ \\
\hline & $\begin{array}{l}\text { 2) Eigen-components may not be } \\
\text { representative if slow-time ensembles are not } \\
\text { spatially stationary }\end{array}$ & $\begin{array}{l}\text { 2) Eigen-components must take } \\
\text { on the form of complex sinusoids }\end{array}$ \\
\hline $\begin{array}{l}\text { Preferred application } \\
\text { scenarios }\end{array}$ & $\begin{array}{l}\text { 1) Imaging cases with highly accelerative } \\
\text { tissue motion }\end{array}$ & $\begin{array}{l}\text { 1) Imaging cases with highly } \\
\text { spatially-varying tissue motion }\end{array}$ \\
\hline & $\begin{array}{l}\text { 2) Peripheral flow imaging with fine spatial } \\
\text { resolution }\end{array}$ & $\begin{array}{l}\text { 2) Deep-vessel imaging with } \\
\text { coarse spatial resolution }\end{array}$ \\
\hline
\end{tabular}

tistics, and they may take on arbitrary shapes because no statistical assumption is made about the data stationarity in the slow-time domain. Nevertheless, it may not be straightforward to define spatial windows whose slow-time ensembles are statistically stationary, especially when the CFI sample volume of interest is positioned near vessel walls or myocardium that may induce local tissue motion over the cardiac cycle. In view of these attributes, the multi-ensemble approach seems more suitable for CFI scenarios with highly-accelerative tissue motion or in peripheral CFI scenarios where tissue motion is more likely to be uniform over the limited depth-of-view.

In contrast, as its name implies, the single-ensemble eigen-estimation approach computes eigen-components using only the slow-time ensemble at the CFI sample volume of interest. This formulation is based on the formation of Hankel data matrices from lag-one overlapping subsets of the slow-time ensemble, so the resulting eigen-components are essentially estimated from an intra-ensemble average of slow-time data samples. From an application standpoint, the single-ensemble approach is versatile in the sense that it can be applied to any slow-time ensemble of interest without needing to define a spatial window with stationary clutter characteristics. Hence, it can be useful in CFI scenarios with highly spatially-varying tissue motion (e.g., transthoracic coronary imaging). Perhaps an additional advantage of the single-ensemble approach is that the filtered power and velocity estimates can be obtained directly from the eigen-component characteristics without having to reconstruct the filtered ensemble. In terms of its disadvantages, the single-ensemble approach can provide at most half the number of eigen-components as can be obtained form the multi-ensemble formulation, and these eigen-components are limited to the form of complex sinusoids because slow-time data stationarity is assumed when forming the Hankel matrix. These limita- tions inherently make the single-ensemble approach less effective in representing clutter that span a wide Doppler spectral band.

In terms of their computational demand, both eigenestimation approaches are rather burdensome. In particular, the multi-ensemble approach requires on the order of $N_{D}^{3}$ flops per eigen-estimation [14], whereas the single-ensemble approach needs on the order of $P^{3}$ flops [15]. As illustrated in [13], eigen-based filters are typically more computation-intensive than non-adaptive clutter filters, even though real-time implementations can still be achieved with the use of efficient computation methods. Indeed, with recent advances in computation hardware such as graphical processing units [19], the additional computational expense in using eigen-based clutter filters should become even more affordable.

\section{The Second Stage of Eigen-Filters: Filter Order Selection}

\section{A. General Overview}

For the eigen-based clutter filter to perform properly, it is essential to choose a suitable filter order whose attenuation response can adequately suppress clutter without distorting the desired blood echoes in the slow-time ensemble. This filter order selection process essentially involves identifying which of the estimated eigen-components are representing clutter. In particular, it requires two quantities to be determined: 1) the number of eigen-components corresponding to clutter, $K_{\mathrm{c}}$, and 2) the clutter eigen-component subset $\boldsymbol{\Phi}_{\mathrm{c}}$.

The challenge in selecting the correct eigen-filter order is that the slow-time signal characteristics often leverage how the eigen-components are distributed over the con- 


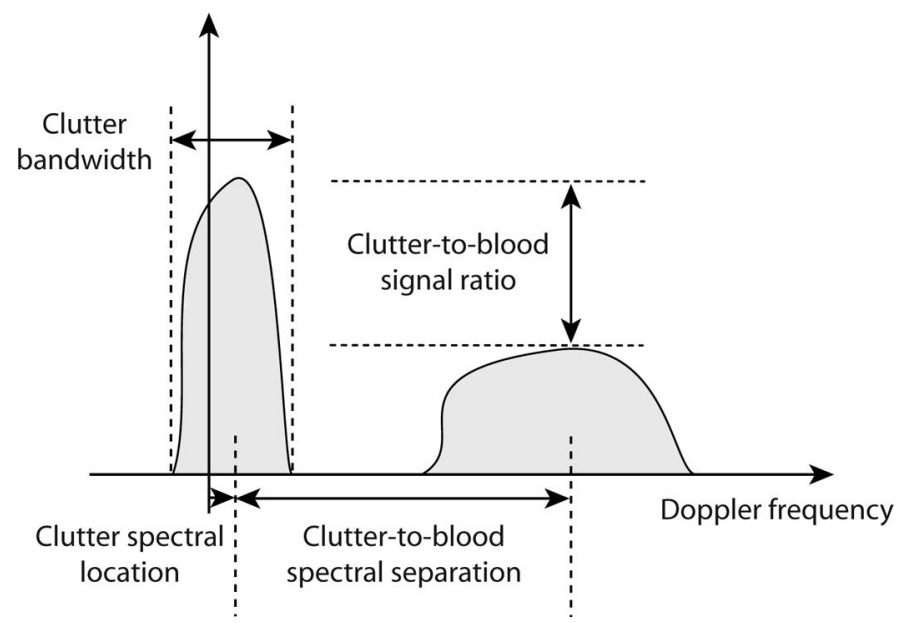

Fig. 3. Doppler spectral features that influence filter order selection. Important parameters include the clutter-to-blood signal ratio (CBR), the clutter-to-blood spectral separation (CBSS), and the clutter spectrum's bandwidth and spectral location.

stituent components (i.e., blood echoes, clutter, or noise floor). From a frequency perspective, as shown in Fig. 3 , there are at least four Doppler spectral features that influence the eigen-component distribution: the clutterto-blood signal ratio (CBR), the clutter-to-blood spectral separation (CBSS), and the clutter component's center Doppler frequency $f_{c}$ and bandwidth $B_{c}$. It is well-accepted that these features vary for different CFI scenarios and also over different phases of the cardiac cycle. For instance, in high-frequency CFI studies, the CBR may actually approach $0 \mathrm{~dB}$ because of the Rayleigh scattering properties of blood. Also, in the microvasculature and during cardiac diastole, the CBSS tends to be narrower because of the slower blood flow. In cases with tissue movement, the center Doppler frequency of clutter may drift away from zero. Indeed, when the tissue motion is accelerative, the clutter bandwidth generally becomes larger.

These Doppler spectral features may pose various complications for the eigen-filter order selection process. First, when the CBR is small, the more dominant eigencomponents tend to be distributed intermittently over the clutter and blood constituents, thereby making it more challenging to identify the clutter eigen-components. Complication may also arise when the CBSS is narrow, because in this scenario, some of the eigen-components may span both the clutter and blood constituents. A further complication may occur when the clutter bandwidth is large, because more of the estimated eigen-components would be related to clutter. Note that the latter two complications generally become more significant when a longer ensemble period is used to acquire the slow-time ensembles (e.g., by lowering PRF or by increasing ensemble size $N_{D}$ ), because of the finer Doppler spectral resolution that results.

In view of the challenges in eigen-filter order selection, some studies have proposed algorithms for selecting clutter eigen-components based on the information available in both the eigenvalue spectrum [10], [13] and in the frequency content of individual eigenvectors [15]. The concepts and potential pitfalls of these algorithms will be further described in the following subsections. It is worth noting that a few array-processing-based order selection strategies like minimal description length and Akaike's information criterion have previously been applied to CFI data processing too [4]. However, they are known to underestimate the number of clutter eigen-components (as shown in an eigen-based flow estimator study [20]), so they will not be further considered here.

\section{B. Eigenvalue-Based Algorithms}

It is well-known that eigenvalues contain information about the signal energy represented by the individual eigen-components. In particular, a large eigenvalue signifies that its respective eigen-component accounts for a substantial energy portion of the slow-time ensemble. Such a representation often matches with the characteristics of clutter, which usually has higher power than the other constituent components because of tissues' stronger scattering strength. Indeed, it was the basis for early eigen-filter designs that defined their attenuation response by setting the clutter eigen-component subset as a fixed number of the most dominant eigen-components [6]-[9], [11].

An algorithmic way of selecting eigen-filter orders based on eigenvalue information is to identify the clutter eigencomponents as the ones whose respective eigenvalues are above a predefined clutter energy value $\lambda_{c}[10]$. This algorithm may enhance the eigen-filtering performance in cases where clutter does not span the same number of eigen-components over time and at different CFI sample volumes. Note that the resulting clutter eigen-component subset $\boldsymbol{\Phi}_{\mathrm{c}}$ being identified can be expressed as

$$
\boldsymbol{\Phi}_{\mathrm{c}}=\left\{\mathbf{e}_{k} \mid \lambda_{k}>\lambda_{\mathrm{c}}\right\}
$$

where the threshold can, for instance, be set relative to the most dominant eigenvalue. Aside from direct thresolding of eigenvalues, the clutter eigen-component subset may also be chosen based on the relative distribution between eigenvalues: more specifically, by thresholding the eigenvalue differences $\lambda_{k}-\lambda_{k+1}$ or ratios $\lambda_{k} / \lambda_{k+1}$ [13]. The rationale for using these latter strategies is that the eigenvalue spectrum would likely flatten once higher bandwidth signals such as that of blood and thermal noise are introduced.

\section{Frequency-Based Algorithms}

By investigating the frequency content of the individual eigenvectors, information can be obtained about the Doppler spectral band that each eigen-component represents. One frequency measure that is of interest is each eigenvector's mean Doppler frequency $f_{k}$, and it can be estimated using the lag-one autocorrelation formula as given by [18] 


$$
\begin{aligned}
f_{k}= & \frac{\mathrm{PRF}}{2 \pi} \arg \left\{R(1)_{k}\right\} \\
& \quad \text { for } R(1)_{k}=\frac{1}{N_{D}-1} \sum_{m=0}^{N_{D}-2} e_{k}^{*}(m) e_{k}(m+1),
\end{aligned}
$$

where $R(1)_{k}$ represents the lag-one autocorrelation value for the $k$ th eigenvector. With these frequency estimates, we may identify the clutter eigen-components as the ones whose mean frequency lies within a bandwidth $B_{\mathrm{c}}$ that is centered around the center clutter frequency $f_{\mathrm{c}}[15]$. The resulting clutter eigen-component subset $\boldsymbol{\Phi}_{\mathrm{c}}$ can then be expressed as

$$
\mathbf{\Phi}_{\mathrm{c}}=\left\{e_{k}|| f_{k}-f_{\mathrm{c}} \mid<\frac{B_{\mathrm{c}}}{2}\right\}
$$

In practice, the mean Doppler clutter frequency $f_{\mathrm{c}}$ in (15) can be assumed to be zero if the tissue movement is small compared with that of blood flow. Alternatively, this quantity can be set equal to the mean frequency of the most dominant eigen-component (i.e., $f_{\mathrm{c}}=f_{1}$ ) if the CBR is known to be high. Such an approach may help to compensate for cases where the clutter spectrum drifts away from zero frequency (which occurs when there is substantial tissue movement).

\section{Comparison of Algorithms}

Perhaps the primary difference between the two filter order selection algorithms is that one assumes clutter to have higher power than blood signals whereas the other assumes clutter to span a Doppler spectral band separable from that of blood signals. These assumptions on clutter characteristics give the eigenvalue-based algorithm the advantage of being able to suppress clutter with very high Doppler frequency contents (arising from very substantial tissue motion), whereas the frequency-based algorithm enjoys the advantage of being able to suppress low-power clutter as long as its Doppler spectrum is separated from that of blood.

Fig. 4 illustrates how the eigen-filter order selection algorithms perform theoretically in three signal scenarios of different Doppler spectral characteristics. The left half of this figure shows the Doppler spectrums and relevant parameters for the three cases, and the right half shows the corresponding eigen-component characteristics by plotting the eigenvalue distribution against the eigenvector mean frequency. For these examples, an ensemble size of 12 samples was used for the slow-time data. The correlation matrix used to compute the eigen-components were computed using the same theoretical model employed in prior clutter filter studies [7].

For the top plots of Fig. 4, a moderate CBR and CBSS is specified, and it can be observed that both eigenvalue- and frequency-thresholding algorithms can effectively isolate the dominant and low-frequency clutter component. As another example, the middle plots show that the eigenvalue-based algorithm can potentially be ineffective when the CBR approaches $0 \mathrm{~dB}$. In this case, it appears that the frequency-based algorithm is more capable of discerning the clutter eigen-components from the other ones. As a further consideration, the bottom plots show a case where the CBSS is relatively narrow. It can be seen that the resulting eigen-components for clutter and blood have similar mean frequencies, hence making it difficult for the frequency-based algorithm to choose a suitable threshold during operation. On the other hand, because the corresponding eigenvalues for the clutter eigen-components are not clustered with those for blood, the eigenvalue-based algorithm should still be able to perform properly. These examples suggest that accurate identification of clutter eigen-components may require the combined use of eigenvalue and frequency information.

\section{In Vivo Assessment: Methodology}

\section{A. Overview of Study}

To assess how different eigen-filter formulations influence the clutter suppression performance in practice, we conducted CFI studies for two in vivo cases: 1) intra-operative imaging of coronary arteries in the presence of cardiac contraction, and 2) diagnostic imaging of nodule neovascularization (tiny blood vessels) in the thyroid. These two in vivo cases have been chosen for this work because they are both challenging from a flow-detection standpoint. In particular, the intra-operative coronary imaging example represents a case with spatially and temporally varying clutter caused by substantial tissue motion that arises from cardiac contraction, whereas the thyroid imaging example corresponds to a scenario with high susceptibility to minor tissue motion because of the use of a low PRF (i.e., high Doppler resolution) for slow flow detection. For both examples, the CBSS can be small or even approach zero, so clutter has a high chance of leaking through conventional high-pass filters and consequently leading to flashing artifacts as well as biased velocity estimates.

\section{B. Analysis Procedure}

In this study, multiple frames of raw CFI data were recorded using a Vivid-7 ultrasound system (GE Vingmed Ultrasound, Horten, Norway) that has been configured according to the settings listed in Table II. With these raw data sets, we then analyzed the performance of eigen-based clutter filters that involve: 1) multi-ensemble eigen-estimation based on a $10 \times 10$ window of independent slow-time ensembles; 2) multi-ensemble eigen-estimation based on $5 \times 5$ windows; 3 ) single-ensemble eigen-estimation. For each form of eigen-filter, we examined the efficacy of both eigenvalue-based and frequency-based filter order selection algorithms, but in this paper we only present results obtained using the frequency-based algorithm because it 
Doppler scenario 1

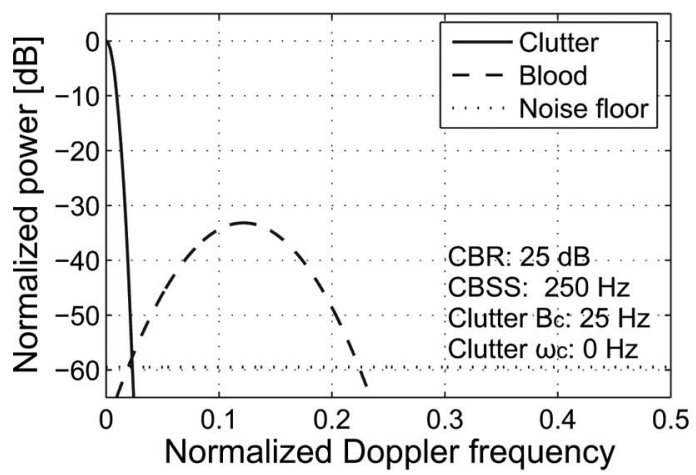

Doppler scenario 2

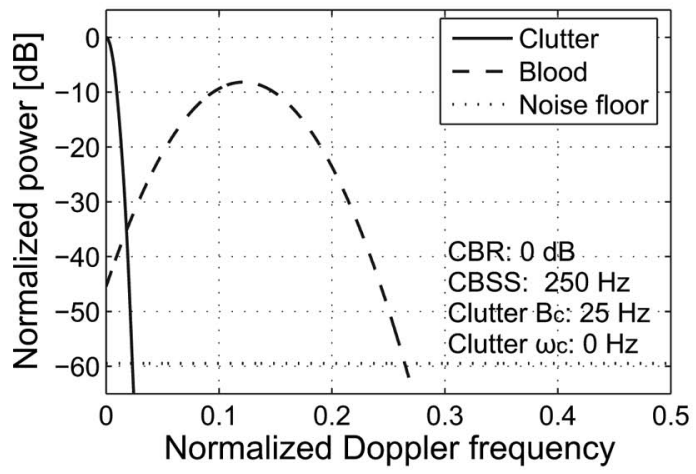

Doppler scenario 3

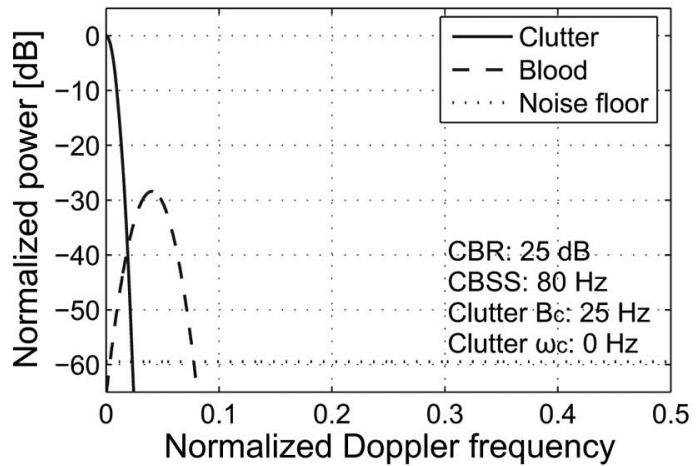

Eigenvalue power vs. mean frequency

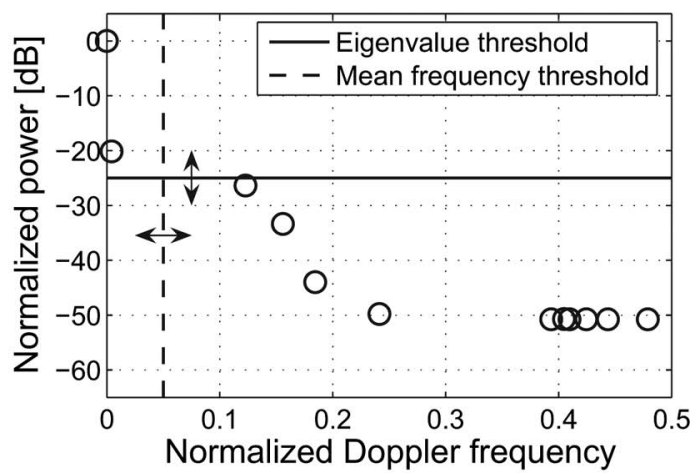

Eigenvalue power vs. mean frequency

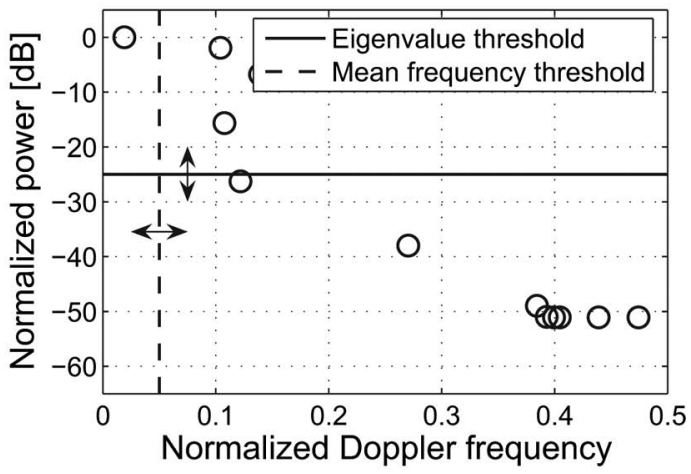

Eigenvalue power vs. mean frequency

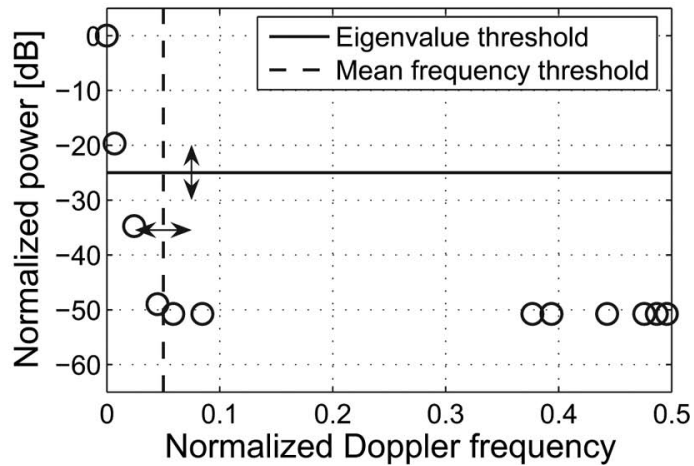

Fig. 4. Concepts and pitfalls of different filter order selection algorithms. The left and right columns respectively show the theoretical Doppler spectrum and the eigen-component characteristics. Three signal scenarios are considered: $\mathrm{CBR}=25 \mathrm{~dB}$ and $\mathrm{CBSS}=250 \mathrm{~Hz}$ (top row); $\mathrm{CBR}=0 \mathrm{~dB}$ and $\mathrm{CBSS}=250 \mathrm{~Hz}$ (middle row); $\mathrm{CBR}=25 \mathrm{~dB}$ and $\mathrm{CBSS}=80 \mathrm{~Hz}$ (bottom row).

was apparently not possible to achieve consistent filtering results using the eigenvalue-based algorithm [21]. It should be emphasized that, in our analysis, the eigen-estimation process was carried out separately for the raw slow-time ensemble of every CFI sample volume within the imaging view. For the multi-ensemble eigen-estimation approach, the spatial window was set to be centered around the CFI sample volume concerned.

The eigen-based clutter filters' performance was qualitatively assessed by examining the flow visualization performance of the Doppler power maps formed from the filtered signal power estimates. We also evaluated the filtering performance quantitatively in two ways: 1) by studying the distribution of eigenvalues and eigenvector mean frequencies from two regions of interest (ROIs) that
TABLE II. Acquisition Parameters Used in the IN Vivo Imaging Studies.

\begin{tabular}{lll}
\hline Parameter & $\begin{array}{l}\text { Coronary } \\
\text { imaging }\end{array}$ & $\begin{array}{l}\text { Thyroid } \\
\text { imaging }\end{array}$ \\
\hline Probe type & GE i13L & GE M12L \\
Pulse center frequency & $10 \mathrm{MHz}$ & $6 \mathrm{MHz}$ \\
F-number (Tx/Rx) & $1.4 / 1.1$ & $2.5 / 1.4$ \\
Lateral beam width & $0.17 \mathrm{~mm}$ & $0.36 \mathrm{~mm}$ \\
Axial range of sample volume & $0.16 \mathrm{~mm}$ & $0.20 \mathrm{~mm}$ \\
Pulse repetition frequency & $2500 \mathrm{~Hz}$ & $350 \mathrm{~Hz}$ \\
Slow-time ensemble size & 10 & 12 \\
\hline
\end{tabular}



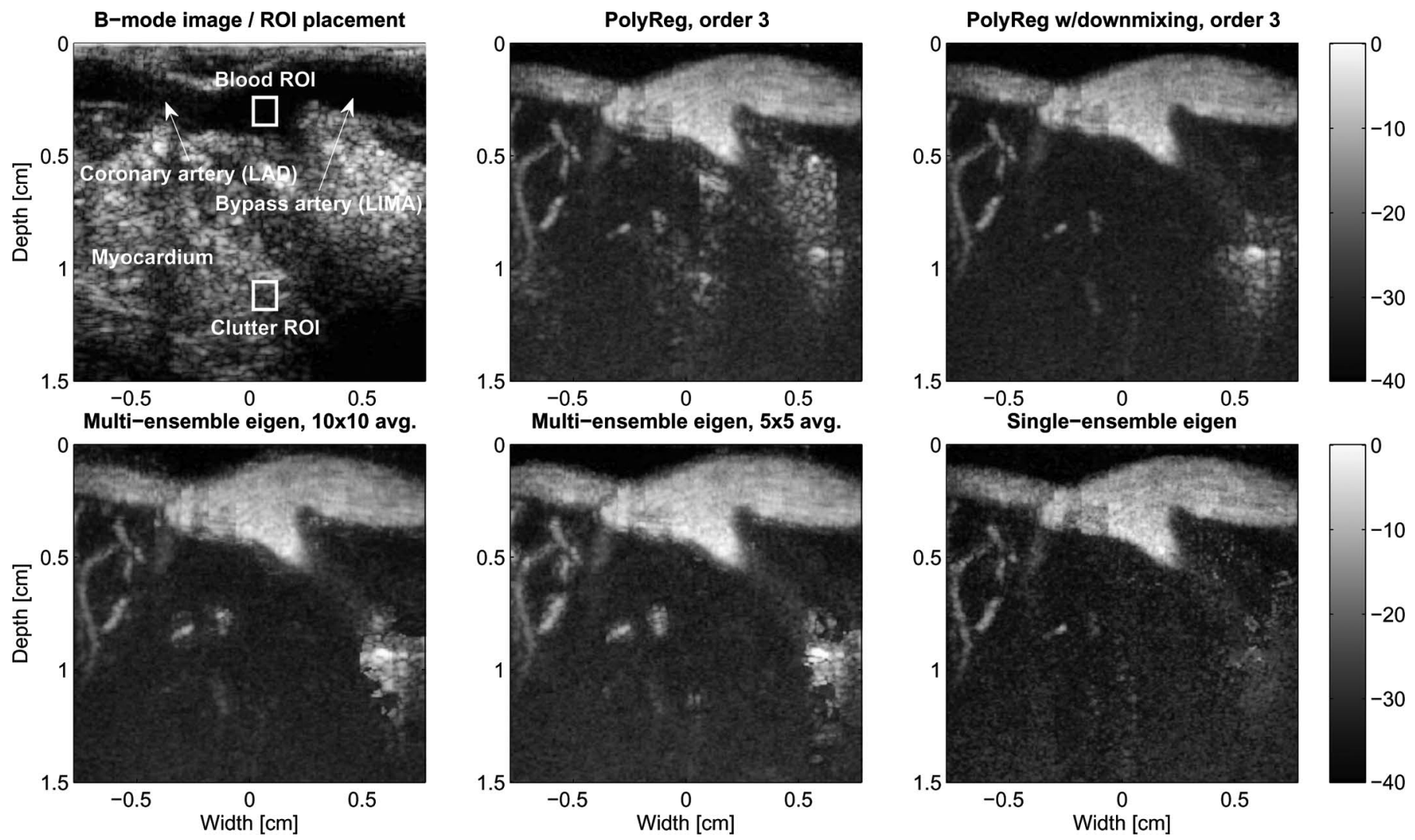

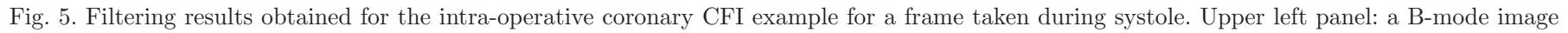
showing the location of the native coronary and bypass artery, as well as the ROIs used in the inter-frame and eigen-distribution analysis. Remain-

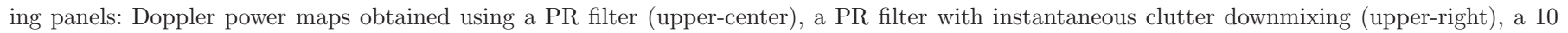

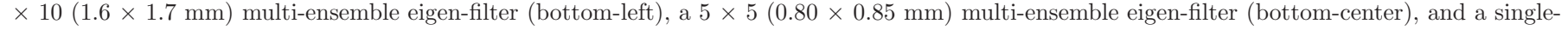

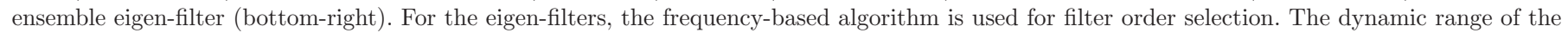
power map scale is $40 \mathrm{~dB}$.

respectively encompass tissues and blood vessels; 2) by measuring the filtered power over various cardiac cycle phases for the same ROIs. To facilitate comparison with existing clutter filters, we repeated the data analysis procedure using polynomial regression (PR) filters that are not adaptive to tissue motion [2] as well as PR filters with instantaneous clutter downmixing [7]. Note that the PR filter order was empirically chosen such that the filter has a nominal cutoff frequency corresponding to the threshold used by the eigen-filters' frequency-based filter order selection algorithm.

\section{In ViVo Assessment: Results}

\section{A. Background of Scenarios}

1) Intra-Operative Coronary Imaging: This type of CFI diagnosis is sometimes carried out during a coronary artery bypass grafting surgery, and in principle it can serve a few clinical purposes [22]. First, it can help verify whether the graft has supplied sufficient blood to ischemic regions of the myocardium. Second, it can be used to assess the suitability of the chosen site for bypass graft placement (i.e., preferably not in the immediate proximity of larger septal or diagonal vessel branches). Third, it can check for whether a stenosis has occurred due to surgical errors. To perform these diagnoses reliably, the CFIs must be capable of providing visualization of the main larger vessels (about $2 \mathrm{~mm}$ in diameter) as well as the smaller septal branches (sub-millimeter in size). However, this imaging requirement is rather challenging to meet over the entire cardiac cycle because of the significantly varying coronary blood flow. In particular, when the heart is contracting there is little blood flow, whereas when the heart is relaxing there is a substantial increase in blood flow.

2) Thyroid Imaging: In clinical diagnoses of the thyroid, CFI can help search for potential markers related to the malignancy of suspected tumors. In particular, it is technically possible to use CFI to observe the amount of neovascularization inside the thyroid nodule and to determine whether a halo-like ring-shaped vessel is encapsulating the nodule. Nevertheless, the flow visualization quality of CFI in this diagnostic scenario is often obscured by flashing artifacts that appear because of two sources of motion: 1) probe navigation, and 2) tissue movement originating from patient swallowing and pulsations of the nearby carotid artery. A further complication in performing thyroid CFI diagnoses is that a lower PRF must be used to im- 

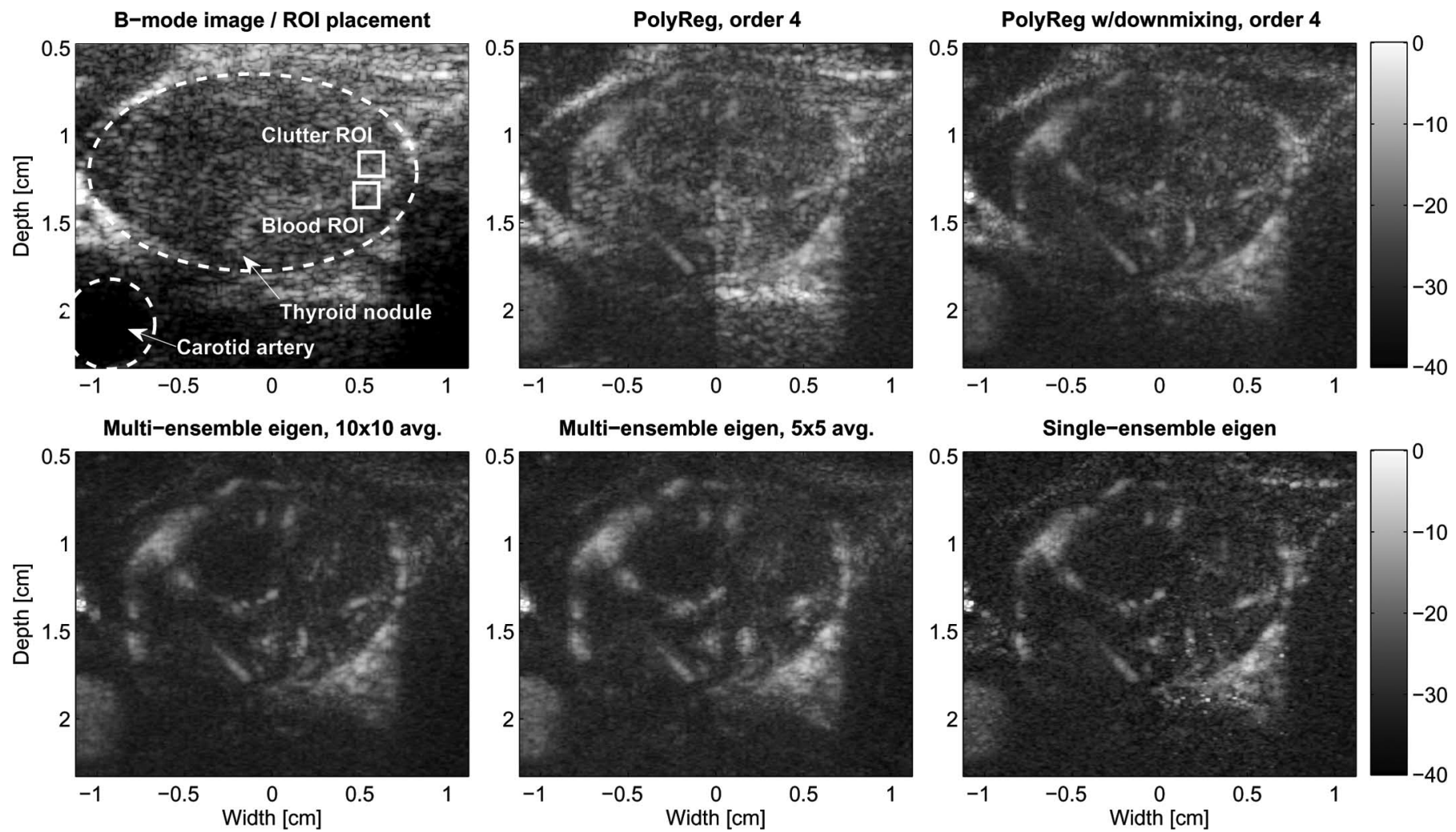

Fig. 6. Filtering results obtained from the thyroid CFI example. Upper-left panel: a B-mode image showing the location of the thyroid nodule and the carotid artery, as well as the ROIs used in the inter-frame and eigen-distribution analysis. Remaining panels: descriptions are the same as Fig. 5. Window dimensions for the multi-ensemble eigen-filters are: $2.0 \times 3.6 \mathrm{~mm}(10 \times 10$ formulation $)$ and $1.0 \times 1.8 \mathrm{~mm}(5 \times 5$ formulation $)$.

prove the Doppler resolution because thyroid blood flow is significantly slower than that in large arteries, and in doing so the CFIs become inherently more prone to flashing artifacts caused by minor tissue motion.

\section{B. Doppler Power Maps}

1) Intra-Operative Coronary Imaging: Fig. 5 shows the filtering results obtained for an imaging frame taken during systole where substantial cardiac motion is present. The upper left panel of this figure is a B-mode image that depicts the location of myocardium, native coronary artery (LAD), and bypass artery (LIMA). The other panels are the Doppler power maps obtained after applying the three forms of eigen-filters being tested (with $250 \mathrm{~Hz}$, or 0.1 normalized frequency, as the clutter frequency threshold) and a 3rd-order PR filter (with and without instantaneous clutter downmixing). Justifications for the choice of clutter frequency threshold used by the eigen-filters will be presented in the next section when we consider the eigencomponent characteristics. Note that the window sizes for the two forms of multi-ensemble eigen-filters being tested are respectively $1.6 \times 1.7 \mathrm{~mm}(10 \times 10$ formulation $)$ and $0.80 \times 0.85 \mathrm{~mm}(5 \times 5$ formulation $)$.

2) Thyroid Imaging: Fig. 6 shows the flow visualization quality of Doppler power maps for the thyroid CFI example. The upper-left panel of this figure is a B-mode image that shows where the thyroid nodule and the carotid artery are located, and the remaining panels are the Doppler power maps obtained using the five clutter filter methods considered in our analysis. Note that for this CFI example, the PR filter order was set to four and the eigen-filter's clutter frequency threshold was set to $50 \mathrm{~Hz}$ (or normalized frequency of 0.143 ). Also, the window size for the two multi-ensemble eigen-filters are $2.0 \times 3.6 \mathrm{~mm}(10 \times 10$ formulation $)$ and $1.0 \times 1.8 \mathrm{~mm}(5 \times 5$ formulation $)$.

3) Discussion: For the intra-operative coronary CFI example (Fig. 5), it can be seen that the PR filter's Doppler power map (upper-center panel) has flashing artifacts in the lower-center and rightmost regions. These artifacts are more effectively suppressed using the PR filter with downmixing (upper-right panel) and the three eigen-filter formulations (bottom panels). Another observation to be noted is that among the eigen-filters, the single-ensemble eigen-filter (bottom right panel) seems to be more capable of suppressing the flashing artifact in the rightmost region, but the small vessels in the lower-center region appear to be concomitantly suppressed. The opposite trend can be observed for the two multi-ensemble eigen-filters: i.e., they are capable of visualizing the lower-center region's blood vessels, but the flashing artifact in the rightmost region remains visible. Note that, for the multi-ensemble eigenfilter, both the $10 \times 10$ formulation (bottom-left panel) and the $5 \times 5$ formulation (bottom-center panel) seem 

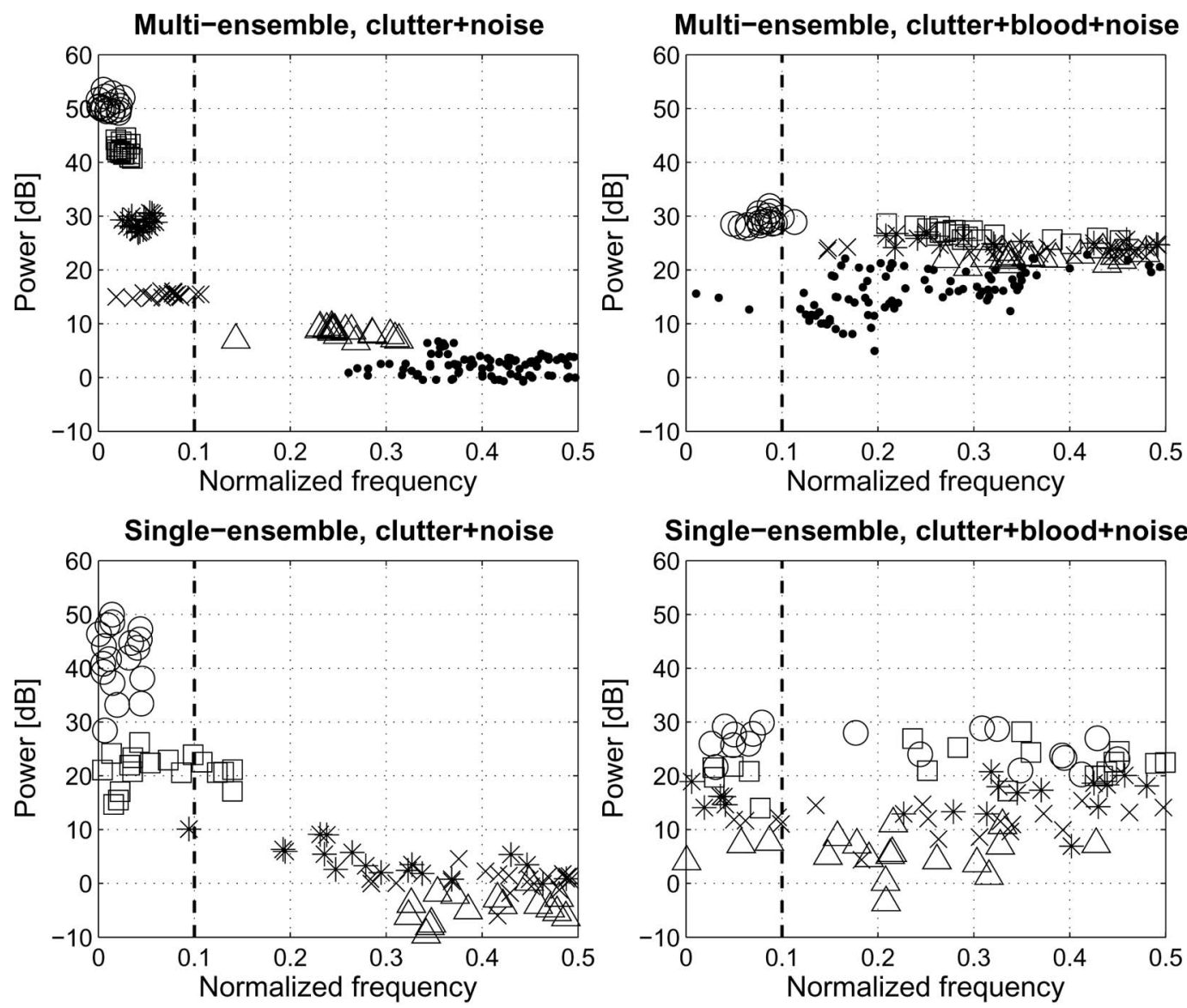

$\bigcirc$ 1st eig $\square$ 2nd eig $*$ 3rd eig $\times$ 4th eig $\triangle$ 5th eig $\bullet$ Remaining eig

Fig. 7. Scatter plots showing the eigen-component characteristics (power versus mean frequency) in the tissue and blood ROIs of the coronary CFI example. Top row: Eigen-component distributions obtained using the $5 \times 5$ multi-ensemble eigen-estimation approach. Bottom row: Results obtained using the single-ensemble eigen-estimation approach. The left and right columns respectively show the results for the tissue ROI and the blood ROI (as marked in the upper-left panel of Fig. 5). The dashed vertical line shows the frequency threshold used to select the filter order in all examples for this case.

to yield a similar flow visualization quality. This finding suggests that using spatial windows with less than $1.6 \times$ $1.7 \mathrm{~mm}$ dimensions would not affect the performance of the multi-ensemble eigen-filter in this CFI example.

An overall similar trend can be seen for the thyroid CFI example. As shown in Fig. 6, the PR filter's Doppler power map (upper-center panel) contains a flashing artifact in the right half of the image, likely because of inadequate clutter suppression in this region. The downmixing step has helped to reduce the flashing artifact (upper-right panel of Fig. 6), but the tiny vessels inside the thyroid nodule are not as well visualized as those seen from the eigen-filters' Doppler power maps (three bottom panels). Comparing between the three eigen-filters, the single-ensemble eigen-filter (bottom-right panel) seems to offer a sharper visualization of the micro-vasculature within the thyroid. On the other hand, the multi-ensemble eigen-filters produced Doppler power maps (bottom-left and bottom-center panels) with smeared blood regions. As such, in this CFI example, it may not be trivial to define a suitable spatial window size for the multi-ensemble eigenestimation approach.
Both CFI examples have generally confirmed that adaptive clutter filtering can more effectively suppress clutter in scenarios with significant tissue motion (relative to the slow-time sampling rate PRF). In addition, the Doppler power maps suggest that eigen-filters involving frequency-based filter order selection are more capable of distinguishing between blood and tissue regions as compared with the partially adaptive filtering approach involving instantaneous clutter downmixing before PR filtering. Nevertheless, for the three eigen-filter designs examined here, they each seem to have their own shortfalls in suppressing clutter, so they should not be considered as "magic bullet" solutions to the CFI clutter filtering problem.

\section{Eigen-Component Distribution}

1) Intra-Operative Coronary Imaging: As quantitative insights on the eigen-based filtering approach, Fig. 7 shows the mapping between the power and mean frequency of principal eigen-components at every CFI sample volume within the blood and tissue ROIs shown in the upper-left panel of Fig. 5. These scatter plots were formed by com- 

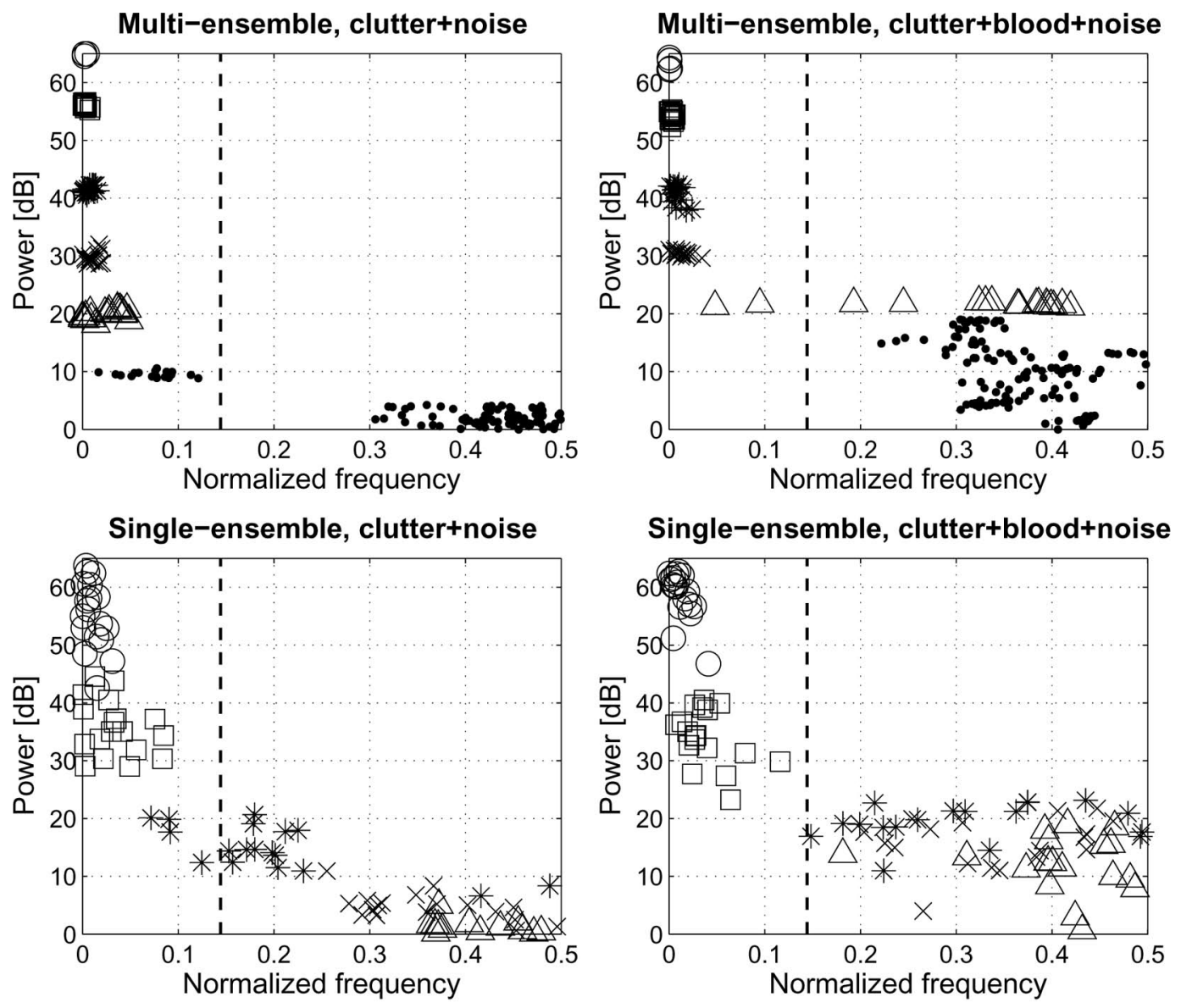

$\bigcirc$ 1st eig $\square$ 2nd eig $*$ 3rd eig $\times$ 4th eig $\triangle$ 5th eig $\bullet$ Remaining eig

Fig. 8. Scatter plots of the eigen-component distribution (power versus mean frequency) for the thyroid CFI example. Descriptions are the same as those of Fig. 7. The results shown correspond to the tissue and blood ROIs indicated in the upper-left panel of Fig. 6.

puting the eigenvalues and the mean frequency of eigenvectors via the single-ensemble and $5 \times 5$ multi-ensemble eigen-estimation methods. Note that, because the singleensemble eigen-estimation method only produces $N_{D} / 2$ eigen-components, its corresponding scatter plots contain fewer markers overall compared with those for the multiensemble eigen-estimation method. It should also be emphasized that these plots merely serve as an illustration of the eigen-component characteristics within two representative ROIs, so their appearance may be different in other parts of the imaging view.

2) Thyroid Imaging: Fig. 8 shows the eigen-component scatter plots for the thyroid CFI example. In general, it should be known that the eigen-component distribution differs between the slow-time ensembles within a tissue region and those within the vasculature. Hence, if the same eigen-filter order is applied to both tissue and blood regions, it is likely that the blood echoes may be concomitantly suppressed (i.e., not desirable for flow detection). This helps explain why an algorithmic way of selecting the eigen-filter order is preferred.
3) Discussion: Several observations important to eigenfilter order selection can be made from the two sets of scatter plots. First, as can be seen for the tissue ROI of both CFI examples (left column of Figs. 7 and 8), the dominant eigen-components of both eigen-estimation approaches are clustered within the low-frequency regime (i.e., to the left of the dashed line in the two plots) and are distinctly higher in Doppler power than the remaining eigen-components. Because the slow-time ensembles within tissues only comprise clutter and noise, those dominant eigen-components are likely to represent clutter. As such, it is reasonable to select the eigen-filter order based on eigenvalue or frequency criterions.

To appreciate why a frequency-based filter order selection algorithm is more suitable for this work, it is necessary to consider the eigen-component characteristics within the blood ROI (see right column of Figs. 7 and 8). In both CFI examples, fewer dominant eigen-components lie within the low-frequency regime, so it appears that clutter is represented by fewer eigen-components (assuming that clutter characteristics in the blood ROI are similar to those seen within the tissue ROI). In addition, for the cor- 
Clutter region post filtering ratio

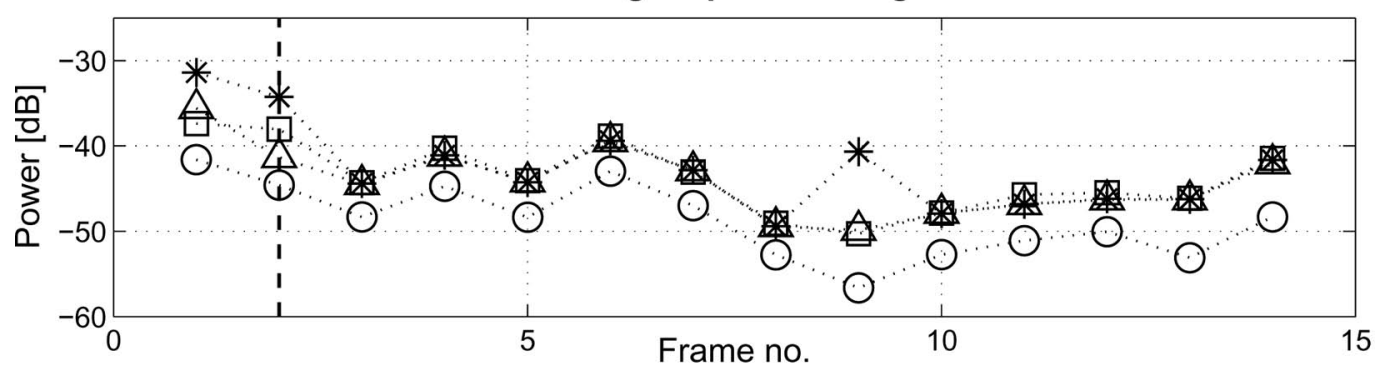

Blood region post filtering ratio

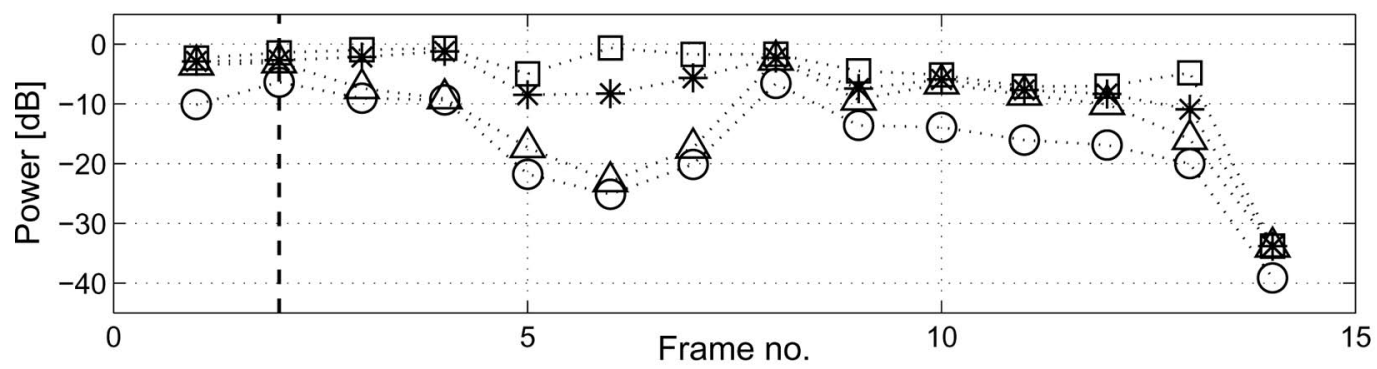

Mean velocity for blood ROI

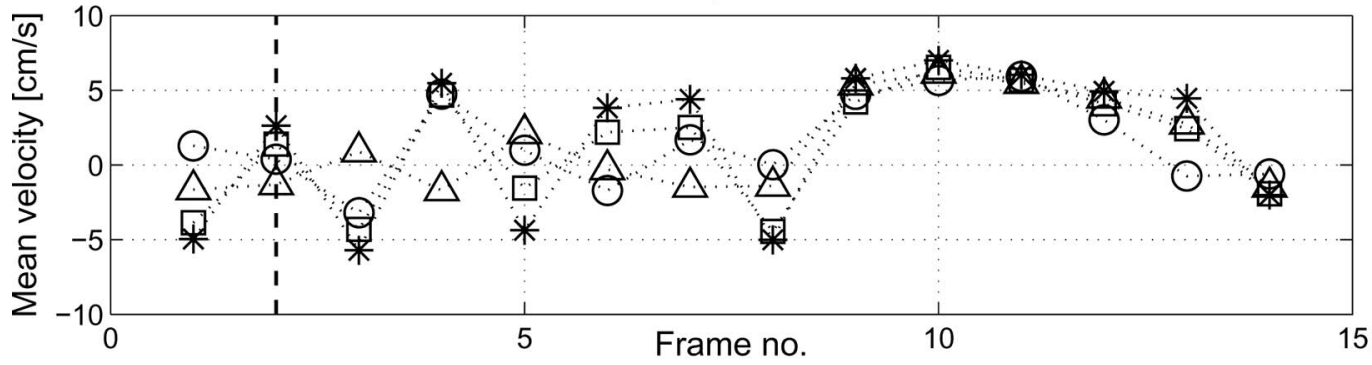

\section{Multi-ensemble $\cdot \bigcirc \cdot$ Single-ensemble $\cdots \triangle$ PolyReg w/downmixing $\cdots *$ * PolyReg}

Fig. 9. Inter-frame analysis of clutter filters for the coronary CFI example, showing filter performance for the blood and tissue ROIs indicated in the upper-left panel of Fig. 5. First two rows: average post-filter Doppler power (with respect to pre-filter power) for the tissue and blood ROIs, shown for the PR filter (with and without downmixing), $5 \times 5$ multi-ensemble eigen-filter, and single-ensemble eigen-filter. Bottom row: average post-filter velocity estimates in the blood ROI, obtained using the lag-one autocorrelation estimator (assuming no angle correction). The dashed line indicates the CFI frame presented in Fig. 5 .

onary CFI example, the largest eigen-component's Doppler power is only slightly higher than that of the others, whereas for the thyroid CFI example, this component's Doppler power remains higher. From these observations, it can be seen that clutter (presumably represented by the dominant eigen-component in this case) has a low Doppler frequency but may not have a distinctly high Doppler power. Thus, to more effectively identify clutter with these eigen-component characteristics, it seems appropriate to use a frequency-based filter order algorithm for the eigen-filters being tested.

Figs. 7 and 8 also provide insights on the eigen-estimation formulation. For the multi-ensemble approach, the major eigen-components for each CFI sample volume (circles and squares within the upper panels) appear to share similar power and frequency contents. This finding shows that, when the multi-ensemble approach is used to perform eigen-estimation, the eigen-filter characteristics tend to change gradually within the ROI, likely be- cause the eigen-components are computed using a spatial window of slow-time ensembles. In contrast, for the single-ensemble approach, there is intrinsically a higher variation in the eigen-component distribution. Its filter characteristics therefore seem to be more spatially localized. Nevertheless, its eigen-estimation variance seems to be concomitantly higher too. This can be explained by recognizing that the single-ensemble approach's correlation matrix is merely formed by an intra-ensemble mean of at most $N_{D} / 2$ time-lagged ensemble subsets, whereas the multi-ensemble approach involves a spatial averaging of $M$ slow-time realizations (with $M \geq N_{D}$ ).

\section{Inter-Frame Analysis of Filter Performance}

1) Intra-Operative Coronary Imaging: As an analysis of the clutter filters' inter-frame dynamics in the coronary CFI example, the first two rows of Fig. 9 show how the average filtered power estimate changes between frames 
Clutter region post filtering ratio

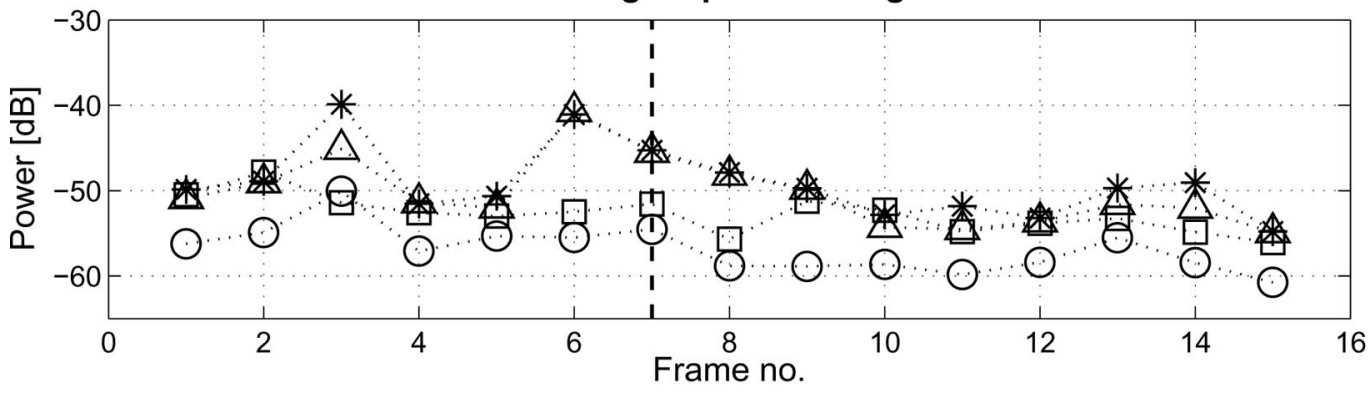

Blood region post filtering ratio

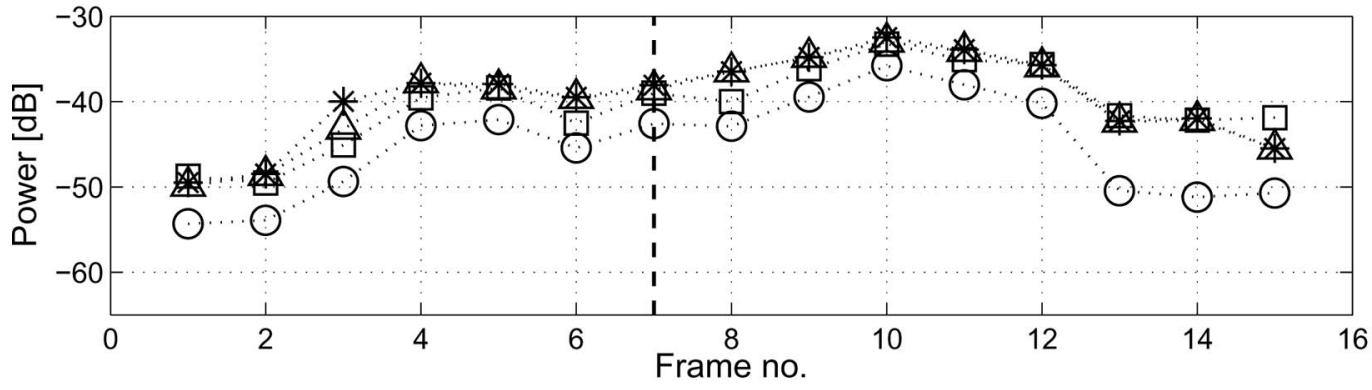

Mean velocity for blood ROI

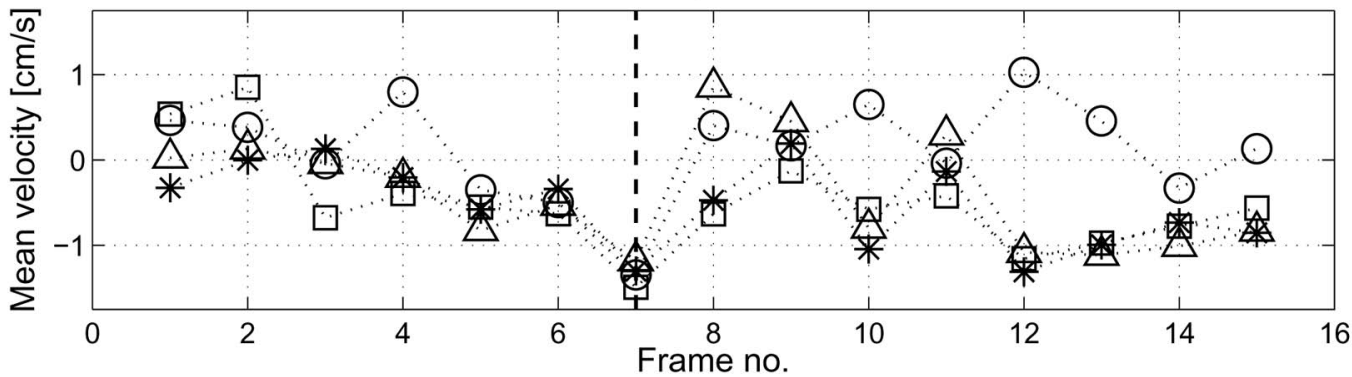

\section{Multi-ensemble $\cdot \bigcirc$. Single-ensemble ' $\triangle$. PolyReg w/downmixing $\cdots *$ * PolyReg}

Fig. 10. Inter-frame dynamics of clutter filters for the thyroid CFI example. The format of this figure is the same as Fig. 9. The tissue and blood ROIs for this CFI example correspond to the marked regions in the upper-left panel of Fig. 6.

for the tissue and blood ROIs labeled in the upper left panel of Fig. 5. These power estimates have been normalized with respect to the raw Doppler power level. Note that the tissue ROI in this case corresponds to a region with substantial tissue motion, as reflected by the flashing artifact seen in the PR filter's Doppler power map in Fig. 5. Also shown in Fig. 9 (bottom row) is the average blood velocity estimates (without angle correction) in the blood ROI over the cardiac cycle.

2) Thyroid Imaging: Fig. 10 shows the corresponding inter-frame dynamics of clutter filters in the thyroid CFI example. Note that the average filtered power level in the blood ROI (middle row) is about $30 \mathrm{~dB}$ lower than that seen for the coronary CFI example. This discrepancy can be explained by realizing that raw slow-time ensembles inside the thyroid vasculature (with sub-millimeter diameters) are likely to have a higher CBR than those inside the coronary arteries (a few millimeters in diameter) where clutter power may be less substantial because tissue lies farther away. Such an explanation is consistent with the scatter plots shown in the right column of Figs. 7 and 8, which show that clutter eigen-components in the thyroid CFI example are higher in Doppler power than the nonclutter ones (whereas clutter and blood echoes' eigen-power level are similar in the coronary CFI example).

3) Discussion: For the tissue ROI of the coronary CFI example (Fig. 9), eigen-based filters (circle- and squaremarked lines) and the PR filter with downmixing (triangle-marked line) produced a better clutter suppression level throughout the cardiac cycle. This observation is consistent with the findings from the Doppler power map analysis. Nevertheless, as can be observed in the middle row of Fig. 9, eigen-based filters may sometimes lead to concomitant suppression of blood signals in the middle of large arteries where clutter power is inherently lower. This finding represents a potential pitfall in using eigen-based clutter filters that choose the filter order via a frequencybased algorithm. The PR filter with instantaneous downmixing seems to offer a balance between eigen-filters and the non-adaptive PR filter (asterisk-marked line). How- 
ever, this type of adaptive filter may lead to velocity estimation biases, as can be seen in the bottom row of Fig. 9 where this filter's blood velocity estimates are sometimes inconsistent with those obtained from the PR filter and the multi-ensemble eigen-filter. It should be noted that the single-ensemble eigen-filter also faces a similar problem in a few CFI frames where the filtered power within the blood ROI is lower for this eigen-filter formulation. Such a result suggests that, among the eigen-filters tested here, the single-ensemble eigen-filter has a higher chance of over-suppressing blood signals, which leads to estimation biases.

The advantages of eigen-based clutter filtering can be better seen in the thyroid CFI example (Fig.10). In particular, compared with the PR filters, eigen-filters exhibited a stronger clutter attenuation response in the tissue ROI over all the CFI frames (see top row), although they did not lead to substantial reduction in the filtered power level within the blood ROI (see middle row). This result shows that eigen-filters have improved the clutter suppression without concurrently reducing the blood signal level. Perhaps one issue of concern is that, the single-ensemble eigen-filter's velocity estimates seem to be biased with reference to the ones obtained after applying other filters (see bottom row). Although we cannot make strong conclusions on this issue without knowledge about the true in vivo flow velocity, the above observation does give indication that the single-ensemble eigen-filter may possibly lead to flow estimation errors caused by difficulties in achieving sufficient clutter attenuation without distorting the blood signal. It may be necessary to develop more accurate flow estimation strategies when using the singleensemble eigen-filter for clutter suppression.

\section{CONCLUding Remarks}

In using eigen-filters for CFI clutter suppression, the eigen-estimation approach and the filter order selection method are two important design issues that need to be considered. In this work, we have presented a comparative assessment of both design issues from theoretical and practical standpoints, and have shown that eigen-filters, if formulated properly, can more effectively suppress clutter in CFI scenarios with substantial tissue motion. Our general conclusion is that although eigen-filters have already demonstrated potential in achieving adaptive clutter suppression, care must be taken to formulate their methodology in order for them to be effective in various Doppler signal scenarios. To improve the adaptiveness of eigenfilters, it may be beneficial to develop an algorithmic way of choosing the eigen-estimation approach and the filter order selection parameters. It may also be worthwhile to pursue the development of theoretically more advanced eigen-estimation methods like independent component analysis so that the clutter and blood eigen-components can be better separated and more easily distinguished during filter order selection. With the improved adaptive- ness, eigen-filters can more effectively address the clutter filtering problem in CFI. In turn, they may improve the overall reliability of flow information provided by CFI and help expand the diagnostic potential of this flow-imaging modality.

\section{Acknowledgments}

We thank R. Cobbold (University of Toronto), P. Cheung (University of Hong Kong), and H. Torp (University of Trondheim, Norway) for their enthusiastic support and encouragement on this work. We would also like to thank K. Helset (Trondheim University Hospital, Norway), S.E. Masoy (University of Trondheim, Norway), and R. Haaverstad (Bergen University Hospital, Norway) for help in providing the in vivo data used in this investigation.

\section{REFERENCES}

[1] H. Torp, "Clutter rejection filters in color flow imaging: A theoretical approach," IEEE Trans. Ultrason. Ferroelectr. Freq. Control, vol. 44, no. 2, pp. 417-424, 1997.

[2] S. Bjaerum, H. Torp, and K. Kristoffersen, "Clutter filter design for ultrasound color flow imaging," IEEE Trans. Ultrason. Ferroelectr. Freq. Control, vol. 49, no. 2, pp. 204-216, 2002.

[3] A. C. H. Yu, K. W. Johnston, and R. S. C. Cobbold, "Frequencybased signal processing in ultrasound color flow imaging," Can. Acoust., vol. 35, no. 2, pp. 11-23, 2007.

[4] M. E. Allam, R. R. Kinnick, and J. F. Greenleaf, "Isomorphism between pulsed-wave Doppler ultrasound and direction of arrival estimation-Part II: Experimental results," IEEE Trans. Ultrason. Ferroelectr. Freq. Control, vol. 43, no. 5, pp. 923-935, 1996.

[5] P. J. Vaitkus, R. S. C. Cobbold, and K. W. Johnston, "A new timedomain narrowband velocity estimation technique for doppler ultrasound flow imaging. Part II: Comparative performance assessment," IEEE Trans. Ultrason. Ferroelectr. Freq. Control, vol. 45, no. 4, pp. 955-971, 1998.

[6] L. A. F. Ledoux, P. J. Brands, and A. P. G. Hoeks, "Reduction of the clutter component in doppler ultrasound signals based on singular value decomposition: A simulation study," Ultrason. Imaging, vol. 19, no. 1, pp. 1-18, 1997.

[7] S. Bjaerum, H. Torp, and K. Kristoffersen, "Clutter filters adapted to tissue motion in ultrasound color flow imaging," IEEE Trans. Ultrason. Ferroelectr. Freq. Control, vol. 49, no. 6, pp. 693-704, 2002.

[8] C. Kargel, G. Hobenreich, B. Trummer, and M. F. Insana, "Adaptive clutter rejection filtering in ultrasonic strain-flow imaging," IEEE Trans. Ultrason. Ferroelectr. Freq. Control, vol. 50, no. 7, pp. 824-835, 2003.

[9] F. Song, D. Zhang, and X. Gong, "Performance evaluation of eigendecomposition-based adaptive clutter filter for color flow imaging," Ultrasonics, vol. 44, pp. e67-e71, 2006.

[10] D. E. Kruse and K. W. Ferrara, "A new high resolution color flow system using an eigendecomposition-based adaptive filter for clutter rejection," IEEE Trans. Ultrason. Ferroelectr. Freq. Control, vol. 49, no. 10, pp. 1384-1399, 2002.

[11] C. M. Gallippi and G. E. Trahey, "Adaptive clutter filtering via blind source separation for two-dimensional ultrasonic blood velocity measurement," Ultrason. Imaging, vol. 24, no. 4, pp. 193-214, 2002.

[12] P. Li, X. Yang, D. Zhang, and Z. Bian, "Adaptive clutter filtering based on sparse component analysis in ultrasound color flow imaging," IEEE Trans. Ultrason. Ferroelectr. Freq. Control, vol. 55, no. 7, pp. $1582-1596,2008$.

[13] L. Løvstakken, S. Bjaerum, K. Kristoffersen, R. Haaverstad, and H. Torp, "Real-time adaptive clutter rejection filtering in color flow imaging using power method iterations," IEEE Trans. Ultrason. Ferroelectr. Freq. Control, vol. 53, no. 9, pp. 1597-1608, 2006.

[14] W. You and Y. Wang, "Adaptive clutter rejection for ultrasound color flow imaging based on recursive eigendecomposition," IEEE 
Trans. Ultrason. Ferroelectr. Freq. Control, vol. 56, no. 10, pp. 2217$2231,2009$.

[15] A. C. H. Yu and R. S. C. Cobbold, "Single-ensemble-based eigenprocessing methods for color flow imaging-Part I. The Hankel-SVD filter," IEEE Trans. Ultrason. Ferroelectr. Freq. Control, vol. 55, no. 3 , pp. 559-572, 2008.

[16] T. K. Moon and W. C. Stirling, Mathematical Methods and Algorithms for Signal Processing. New York, NY: Prentice Hall Inc., 2000, ch. 6-7.

[17] A. C. H. Yu and L. Lovstakken, "Eigen-based clutter filters for color flow imaging: Single-ensemble vs. multi-ensemble approaches," in Proc. IEEE Ultrasonics Symp., 2007, pp. 1101-1104.

[18] C. Kasai, K. Namekawa, A. Koyano, and R. Omoto, "Real-time twodimensional blood flow imaging using an autocorrelation technique," IEEE Trans. Sonics Ultrason., vol. 32, no. 3, pp. 458-464, 1985.

[19] L. W. Chang, K. H. Hsu, and P. C. Li, "Graphics processing unitbased high-frame-rate color Doppler ultrasound processing," IEEE Trans. Ultrason. Ferroelectr. Freq. Control, vol. 56, no. 9, pp. 18561860, 2009.

[20] A. C. H. Yu and R. S. C. Cobbold, "A matrix pencil estimator with adaptive rank selection: Application to in vivo flow studies," in Proc. IEEE Ultrasonics Symp., 2006, pp. 2015-2018.

[21] L. Lovstakken, A. C. H. Yu, and H. Torp, "In vivo investigation of filter order influence in eigen-based clutter filtering for color flow imaging," in Proc. IEEE Ultrasonics Symp., 2007, pp. 2429-2432.

[22] R. Haaverstad, N. Vitale, O. Tjomsland, A. Tromsdal, H. Torp, and S. O. Samstad, "Intraoperative color Doppler ultrasound assessment of LIMA-to-LAD anastomoses in off-pump coronary artery bypass grafting," Ann. Thorac. Surg., vol. 74, no. 4, pp. S1390-S1394, 2002 .

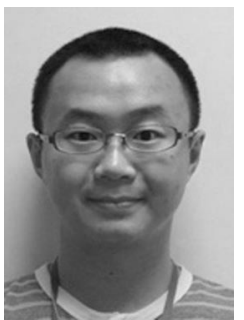

Alfred C. H. Yu was born in Hong Kong in 1980. He received his B.Sc. degree in electrical engineering from the University of Calgary, Calgary, $\mathrm{AB}$, Canada, in 2002. He then completed his M.A.Sc. and Ph.D. degrees in electrical engineering (biomedical stream) from the University of Toronto, Toronto, ON, Canada, in 2003 and 2006, respectively.

In the summer of 2005, he was a research intern at Philips Research, Briarcliff Manor, NY. He is currently a Research Assistant Professor in the Medical Engineering Program and the Department of Electrical and Electronic Engineering at the University of Hong Kong. His research interests are broadly centered on the design aspects of ultrasound systems and devices. He is also interested in studying the fundamentals and physics of ultrasound.

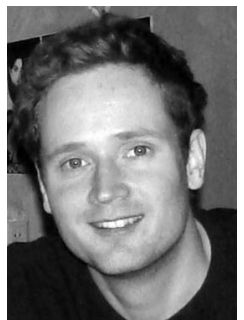

Lasse Lovstakken was born in Bergen, Norway, in 1976. He received his M.Sc. degree in engineering cybernetics and his Ph.D. degree in medical technology from the Norwegian University of Science and Technology in Trondheim in 2002 and 2007, respectively. He is currently working as a Research Fellow at the Department of Circulation and Medical Imaging, at the Norwegian University of Science and Technology. His research interests include signal and image processing with applications in ultrasound imaging. 